\title{
Calculation and Design of Dry-type Air-core Reactor
}

\author{
Yan $\mathrm{Li}^{1}$, Zhenhai Zhang ${ }^{1}$, Longnv $\mathrm{Li}^{1}$,Guoli $\mathrm{Li}^{1}$, Manhua Jiang ${ }^{2}$ \\ ${ }^{1}$ Research Institute of Special Electrical Machines, Shenyang University of Technology, Shenyang, China \\ ${ }^{2}$ Tebian Electric Apparatus Shenyang Transformer Group Co., Ltd., Shenyang, China \\ Email: hailong198759@126.com
}

Received March, 2013

\begin{abstract}
Based on the method of compound and additional conditions under the conditions of the equal temperature rise and the equal potential drop (P.D.) of resistance, the application of design software of dry-type air-core reactor is introduced in this thesis. The analytical methods of the inductance are also given. This approach is proved entirely feasible in theory through the simplification with Bartky transformation, and is able to quickly and accurately calculate reactor inductance. This paper presents the analytical methods of the loss of dry-type air-core reactor as well.
\end{abstract}

Keywords: Dry-type Air-core Reactor; Bartky Transformation; Compound and Additional Conditions; Software Design

\section{Introduction}

With the advantages of light weight, good linearity, high mechanical strength, low noise and easy maintenance, dry-type air-core reactor is a equipment widely used for the current limit, voltage regulator and reactive power compensation in power system [1].

The factors, such as inductance, temperature rise, height and others, should be considered comprehensively in the design of dry-type air-core reactor. The mutual affection and restraint of various factors make the design of dry-type air-core reactor more complicated. In order to simplify the design and improve the efficiency, it is necessary to take appropriate simplification and process. The design adopts the simplified processing mathematical model which is based on the additional constraints. And this paper developed the design software of dry-type air-core reactor.

\section{Calculation Methods}

In the premise of known voltage, capacity, radius and height conditions, then the design of the dry-type air-core reactor could obtain parameters of turns, inductance, temperature rise, current, etc. by using relevant theorems and formulas. It is difficult to directly calculate the outcome. Because of the shortage of given conditions. Thus it's necessary to simplify it without affecting its accuracy. The encapsulating width of the reactor is so far smaller than the coil height that the reactor is considered as the ideal solenoid in the calculation of the inductor. The resistance is much smaller than the inductance of the reac- tor, so that wire could be considered as an ideal conductor, which means we can ignore the affection of resistance. To make the design to meet certain characteristics, such as making the reactor have a minimum temperature rise, minimum supplies, minimum loss, simple structure and convenient manufacturing, we can use the additional constraints of equivalent temperature rise, height and resistance drop method, which could not only make the design more reasonable and achieve structure optimization, but also reduce the computing time and improve efficiency. This article uses additional constraints of entrapment between temperature rise, layer winding resistance drop to achieve minimal supplies, losses and temperature rise of reactor.

\subsection{The Calculation of the Self and Mutual Inductances}

The inductance of the reactor is the most important parameter in the dry-type air-core reactor and its calculation accuracy directly affects the accuracy of the other parameters of the reactor, and then affects the performance of the reactor. According to the reference [2], the mutual inductance of any two coils is :

$$
\begin{gathered}
\mathrm{M}=2 \pi \mu_{0}\left(\mathrm{R}_{1} \mathrm{R}_{2}\right)^{3 / 2} \mathrm{n}_{1} \mathrm{n}_{2}\left[C_{i}\left(R_{1}, R_{2}, Z_{1}\right)-\right. \\
C_{i}\left(R_{1}, R_{2}, Z_{1}\right)+C_{i}\left(R_{1}, R_{2}, Z_{3}\right)-C_{i}\left(R_{1}, R_{2}, Z_{4}\right) \\
C_{i}\left(R_{1}, R_{2}, Z\right)=\frac{\sqrt{R_{1} R_{2}}}{2 \pi} \int_{0}^{\pi} \frac{1}{R_{1}^{2}+R_{2}^{2}-2 R_{1} R_{2} \cos \theta} \\
\sqrt{R_{1}^{2}+R_{2}^{2}+Z^{2}-2 R_{1} R_{2} \cos \theta} \sin ^{2} \theta d \theta
\end{gathered}
$$




$$
\left\{\begin{array}{l}
Z_{1}=S+\frac{H_{1}}{2}+\frac{H_{2}}{2} \\
Z_{2}=S+\frac{H_{1}}{2}-\frac{H_{2}}{2} \\
Z_{3}=S-\frac{H_{1}}{2}-\frac{H_{2}}{2} \\
Z_{4}=S-\frac{H_{1}}{2}+\frac{H_{2}}{2}
\end{array}\right.
$$

where $n_{1} 、 n_{2}$ is the number of turns of the two windings per unit length, $R_{1} 、 R_{2}, S$ respectively are the radius、 the distance , the height of the two windings and $\theta$ presents the horizontal angle.

From the above equation, $C_{i}\left(R_{1}, R_{2}, Z\right)$ is only related with the radius, height, and relative position of two windings, but regardless of the number of turns of the windings. After analysis, we can know that $C_{i}\left(R_{1}, R_{2}, Z\right)$ is a elliptic integral and the computation of its direct integral is complex and not easy to achieve. But it could be simplified and then be solved with the Applied Numerical Solution according to the Bartky transformation method proposed in reference [3]. After using simplification and the Bartky Transformation, the expression is:

$$
\begin{aligned}
& C_{B}(A, B, C, q, k)=\frac{2}{\pi} \int_{0}^{\pi / 2}\left[A \sin ^{2} \varphi+B \cos ^{2} \varphi+\right. \\
& \left.C \frac{\sin ^{2} \varphi}{q^{2} \cos ^{2} \varphi+\sin ^{2} \varphi}\right] \frac{d \varphi}{\sqrt{k^{2} \cos ^{2} \varphi+\sin ^{2} \varphi}}
\end{aligned}
$$

where

$$
\begin{aligned}
& q=\frac{R_{1}-R_{2}}{R_{1}+R_{2}}, 0<|q|<1 ; \quad \gamma=\frac{z^{2}}{4 R_{1} R_{2}} ; \\
& A=\sqrt{1-k^{2}}\left[-\gamma+\frac{1}{3\left(1-k^{2}\right)}\right] ; B=-\frac{k^{2}}{3 \sqrt{1-k^{2}}} ; \\
& k=\frac{\left(R_{1}-R_{2}\right)^{2}+Z^{2}}{\left(R_{1}+R_{2}\right)^{2}+Z^{2}} ; \quad C=\gamma \sqrt{1-k} ;
\end{aligned}
$$

when $R_{1}=R_{2}$, namely $\mathrm{q}=0$, the parameters need to be adjusted to $A=A+C ; B=B+C ; C=0$.

By the Bartky transformation method, the complicated elliptic integral could be simplified to be easily solved with a more accurate calculation.

\subsection{The Calculation of the Encapsulation}

$$
\left\{\begin{array}{c}
j \omega L_{1} \dot{I}_{1}+j \omega M_{12} \dot{I}_{2}+\ldots \ldots \ldots \ldots \ldots \ldots+j \omega M_{1 m} \dot{I}_{m}+R_{1} \dot{I}_{1}=\dot{U}_{N} \\
\vdots \\
j \omega M_{i 1} \dot{I}_{1}+j \omega M_{i 2} \dot{I}_{2}+\ldots . . j \omega L_{i} \dot{I}_{i} \ldots .+j \omega M_{i m} \dot{I}_{m}+R_{i} \dot{I}_{i}=\dot{U}_{N} \\
\vdots \\
j \omega L_{m 1} \dot{I}_{1}+j \omega M_{m 2} \dot{I}_{2}+\ldots \ldots \ldots \ldots \ldots . . j \omega L_{m} \dot{I}_{m}+R_{m} \dot{I}_{m}=\dot{U}_{N}
\end{array}\right.
$$

Suppose the number of encapsulations in reactor is $\mathrm{m}$, and regard each encapsulation as a coil. Set $L_{i}$ as the self-inductance of the ith encapsulation, $M_{i j}$ as the mutual inductance of the ith and jth encapsulątion, $R_{i}$ as the resistance of the ith encapsulation and $I_{i}$ as AC current of the ith encapsulation and $U_{N}$ as the voltage of both ends of reactor. With the basic principle of the circuit, it is possible to calculate the equivalent height, turns number and current of the encapsulation, and then obtain other parameters.

Under general conditions, the resistance is much smaller than the inductor. In the case of ignoring encapsulation resistor, the equation can be equivalent to the following.

$$
\sum_{j=1}^{m} W_{i} W_{j} f_{i j} I_{j}=\frac{U_{N}}{\omega}(i=1,2,3 \cdots, m)
$$

when solving the number of turns, equation 6 is a nonlinear equation. It is difficult to directly calculate, but we can take advantage of some simplifications under certain constraints. Under the conditions of the equivalent temperature rise, we can get the following equation [4].

$$
\begin{gathered}
I_{i}=\frac{a_{i} \sum_{j=1}^{m} a_{j} f_{i j}}{\sum_{k=1}^{m}\left(a_{k} \sum_{j=1}^{m} a_{j} f_{k j}\right)} I_{N} \\
W_{i}=\sqrt{\frac{U_{N}}{\omega I_{N}} \frac{\sum_{k=1}^{m}\left(a_{k} \sum_{j=1}^{m} a_{j} f_{k j}\right)}{\left(\sum_{j=1}^{m} a_{j} f_{i j}\right)^{2}}} \\
=\frac{1}{\sum_{j=1}^{m} a_{j} f_{i j}} \sqrt{L_{N} \sum_{k=1}^{m}\left(a_{k} \sum_{j=1}^{m} a_{j} f_{k j}\right)} \\
\sum_{k=1}^{m}\left(a_{k} \sum_{j=1}^{m} a_{j} f_{k j}\right) \geq \frac{S_{N}}{\omega}\left(\frac{k_{p} \rho J_{i}}{k_{i} H_{i}}\right)^{2}\left(\frac{0.36}{\theta_{\max }}\right)
\end{gathered}
$$

where $L_{N}=\frac{U_{N}}{\omega I_{N}}$ is the rated inductance, $S_{N}$ is the rated capacity and $\theta_{\max }$ is the maximum allowable temperature rise.

From the above equation, we can see that in some cases of ignoring the resistor, nonlinear equations could be converted into linear equations to solve through the additional conditions of the equivalent temperature rise, which can greatly simplify the solution process. Under the condition of knowing the height of dry hollow reactor, encapsulation thickness, the calculated inductance and the maximum temperature of encapsulation, the minimum encapsulation number and the turns number of the respective encapsulating and current, which satisfy the 
requirement, could be calculated. The temperature rise and loss of the encapsulation can be determined after determining the encapsulation turns and current numbers, and then some parameters, such as AWG and the height and width of encapsulation, could be confirmed.

\subsection{Calculation of the Layer Current and the Yurns Number}

The calculation of the layer current and the turns number is similar to the calculation of the encapsulation. If we regard each layer as a coil, its layer equations are nonlinear known from the circuit principle. The resistance is so much smaller than the inductance that the pressure drop of the resistance could be ignored. Thus it's unnecessary to solve nonlinear equations, but directly obtain the number of turns, the current, inductance, thickness and loss parameters of each layer under the constraints of resistance drop method.

Under the constraints of resistance drop method, each layer current and turns number can be obtained as following:

$$
\begin{gathered}
I_{i}=\frac{\sum_{j=1}^{n} \frac{s_{c i} s_{c j}}{r_{i} r_{j}} f_{i j}}{\sum_{k=1}^{n} \sum_{j=1}^{n} \frac{s_{c k} s_{c j}}{r_{k} r_{j}} f_{k j}} I_{N} \\
W_{i}=\frac{\sqrt{L_{N} \sum_{k=1}^{n} \sum_{j=1}^{n} \frac{s_{c k} S_{c j}}{r_{k} r_{j}} f_{k j}}}{\sum_{j=1}^{n} \frac{s_{c j}}{r_{j}} f_{i j}}
\end{gathered}
$$

Where $s_{c i}$ is the conductor sectional area of the ith layer of the coil and $r_{i}$ is the radius distance between the ith layer and the axis.

From the above equation, we can see that by the way of ignoring the resistor and through the additional conditions of the temperature rise, nonlinear equations could be converted into linear equations to solve, which can greatly simplify the solution process. Under the condition of knowing AWG, radius, calculate the inductance and rated current, the turns number and current of each layer, which satisfy the requirement, could be calculated. Then other parameters can be obtained, such as the resistor, the current density, inductance of each layer.

\section{Software Design}

The idea for design is as following: According to the known basic parameters of known, Bartky transformation is applied to solve the mutual inductance coefficient $f_{i j}$. Under the constraint condition of the encapsulation equivalent temperature rise, we can get the encapsulation number meeting the minimum encapsulating condition according to the equation 8.Then the turn number and current of each encapsulation could be obtained on the basis of the equation 7 and 8. Under the comprehensive constraint condition such as AWG, height, ampere density, encapsulation thickness and equal resistance drop method, the turn number and current of every layer could be obtained by the formula 10,11. And then the wire gauge, axial and radial parallel conductors, encapsulation height and current density can be confirmed.

In the computation of the wire gauge, the current and the turn number of each layer as well as current density, the correct parameters must be chosen to iterate computation and the two results are also supposed to be compared to confirm a reasonable error range for a accurate calculation result. If using the wire gauge of the conductor as iteration parameters, the two results would mutate because of the inconsecutive change of the wire gauge of the conductor, thus there is no guarantee for the convergence of the wire gauge. Therefore, the wire gauge should not be chosen as iteration parameter. If choose the height or thickness of each layer whose change is not obvious in Iteration calculations, the change of the parameters could not be accurately determined, and so the precision of calculation was poor. Consequently, the height or thickness of each layer should not be also chosen as iteration parameter. The current density of the conductor not only changes continuously but also converges well, and its variation in the process of iteration is apparent, thus various parameters can be accurately determined. According to the mentioned above, this paper chooses the current density as the iterative parameter. The program flowchart is shown in Figures 1 (a) and (b).

\section{Example}

From the software interface, we can see that the basic parameters such as rated voltage, rated capacity, rated current, winding height and winding diameter need to be input to the interface. The other options can be default values or modified values, which makes the design more flexible, convenient, and applicable.

For example, A dry-type air-core reactor with the following parameters: rated voltage is $10 \mathrm{kV}$, rated current is $400 \mathrm{~A}$, rated capacity is $185 \mathrm{kvar}$, winding height is $0.9 \mathrm{~m}$, winding diameter is $0.8 \mathrm{~m}$, inductance is 3.7674 $\mathrm{mH}$. The design results are shown in Figure 3.

The design results are presented in Figure 3. in detail, which include encapsulation number, encapsulation loss, temperature rise, the number of axial and radial parallel conductors, wire gauge, the thickness, current density, the number of turns, inductance, the turn number of each layer, inductance, current and height. These parameters can fully meet the requirements of a dry-type air-core reactor. 


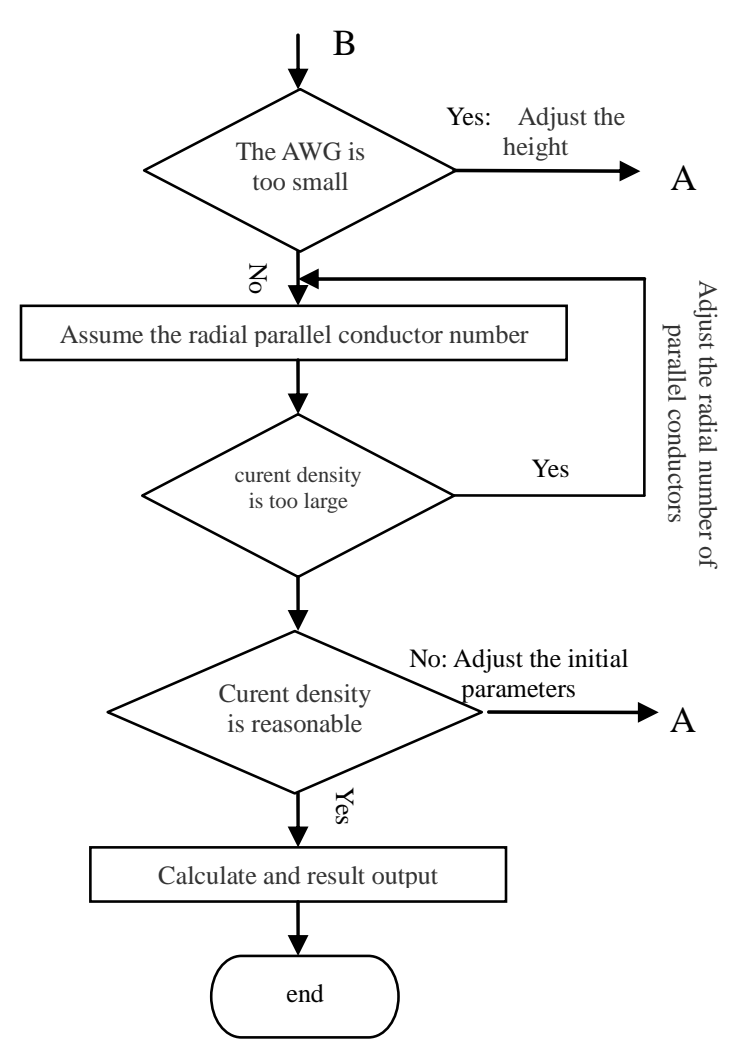

Figure 1. Program flow chart (b).

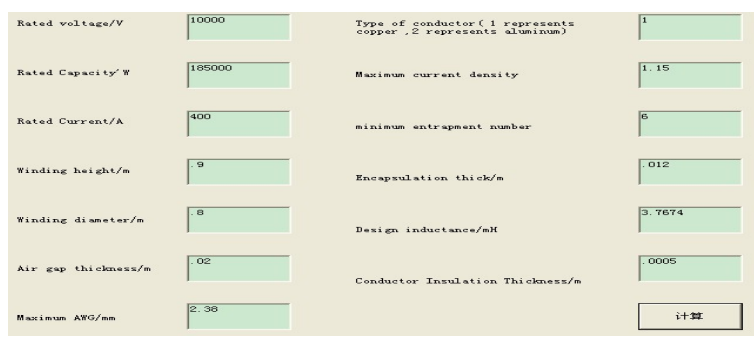

Figure 2. Software interface diagram.

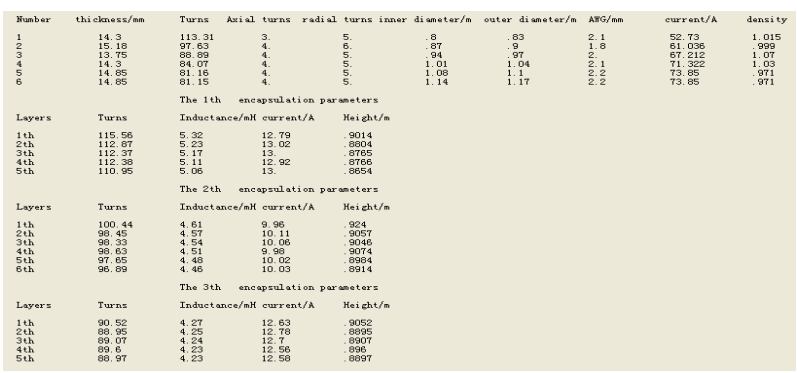

Figure 3. Output result.

\section{Conclusions}

In this paper, the analytical method is used to calculate the inductance of the dry-type air-core reactor, the Bartky transformation is used to simplify it, and the constraints of the temperature rise method and the resistance drop method are used to optimize it. Users only need to enter the basic parameters the design scheme with high accuracy and fast calculation can be obtained. The developed design software interface is simple and easy to use.

\section{REFERENCES}

[1] Z. G. Liu, J. H. Wang and W. P. Wang, "Development and Application of Dry-type Air-core Reactor Design Software," Electric Machines and Control, Vol. 6, No. 7, 2003, pp. 103-106.

[2] X. L. Wei and S. Ma, "Analytic Calculation Method for the Magnetic Field of Air-core Power Reactor with Multi-Layer in Parallel," Transformer, Vol. 2, 1993, pp. 12-15.

[3] T. H. Fawzi and P. E. Burke, "The Accurate Computation of Self and Mutual Inductances of Circular Coils”. IEEE Trans Power Syst. Vol. 97, No. 2, 1978, pp. 464-468.

[4] Z. Min, "Relevant Issues and Computer Aided Design of Hollow Electric Reactor," Harbin: Harbin University of Science and Technology, Mar 2003. 